International Journal of Translational

Medical Research and Public Health (202I),Volume 5, Issue 2, 68-7I

\begin{tabular}{|c|c|c|}
\hline 1 & & $\begin{array}{l}\text { INTERNATIONAL JOURNAL OF TRANSLATIONAL } \\
\text { MEDICAL RESEARCH AND PUBLIC HEALTH } \\
\text { ISSN } 2576-9499 \text { (Online) }\end{array}$ \\
\hline 1]TMRPH & Available online at www.ijtmrph.org & $\begin{array}{l}\text { ISSN 2576-9502 (Print) } \\
\text { DOI: 10.2 I I06/ijtmrph.335 }\end{array}$ \\
\hline
\end{tabular}

\title{
SHORT RESEARCH COMMUNICATION | PEDIATRIC LUNG TRANSPLANT Racial/Ethnic Disparities in Receipt of Lung Transplant and In-Hospital Mortality among Pediatric and Adolescent Patients in the United States
}

\author{
Hamisu M. Salihu, MD, PhD;',2 Brisa Y. Garcia, BS;' Deepa Dongarwar, MS ${ }^{1 凶}$ \\ 'Center of Excellence in Health Equity, Training and Research, Baylor College of Medicine, Houston, Texas, USA; ${ }^{2}$ Department of Family and Community \\ Medicine, Baylor College of Medicine, Houston, Texas, USA \\ Corresponding author email: deepa.dongarwar@bcm.edu
}

\section{ABSTRACT}

The purpose of this study was to analyze racial/ethnic disparity in inpatient mortality among pediatric and adolescent lung transplant recipients ( $\leq 19$ years old) in the United States (US). The Nationwide Inpatient Sample (NIS) dataset from 2003-2017 was used for this analysis. Survey logistic regression model was utilized to find the association between lung transplant and inpatient mortality among various racial/ethnic groups. The overall inpatient mortality rate was 3.92 per 100 lung transplant hospitalizations. Overall, when adjusted for covariates, those who received lung transplants were 8.49 times as likely to experience inpatient mortality (95\% Cl: 5.66-12.73) compared to those who did not receive it. The likelihood of inpatient mortality was highest among Hispanics $(\mathrm{OR}=10.1 \mathrm{I}, 95 \% \mathrm{Cl}=4.93-14.95)$ relative to the findings among other racial/ethnic groups. Our findings may guide healthcare providers when caring for pediatric and adolescent populations from different racial/ethnic backgrounds who need a lung transplant.

Keywords: • Inpatient Mortality • Lung Transplant • Pediatric • Adolescent $\bullet$ Racial Disparity $\bullet$ Nationwide Inpatient Sample

Copyright () 202 I Salihu, et al. Published by Global Health and Education Projects, Inc. This is an open-access article distributed under the terms of the Creative Commons Attribution License CC BY 4.0.

\section{Introduction}

Lung transplant procedures have increased in the entire population over the previous decades in the United States (US). Compared to 2013, there was a $31 \%$ increase in total lung transplant procedures performed in the US in 2018.' Previous studies have analyzed the impact of race/ethnicity on the outcome of adult lung transplant recipients. From 2005-2017, the overall index hospitalization mortality was $5.4 \%$ in adult lung transplant recipients; however, minority adult lung transplant recipients had a higher index hospitalization mortality at $6.5 \%{ }^{2}$ Moreover, in the lung transplant waitlist outcomes from 2005-20I5, Non-Hispanic (NH)-Black, Hispanic, and Asian adult waitlist candidates were less likely to receive a lung transplant than $\mathrm{NH}$-White waitlist candidates; although, there was no difference in waitlist mortality across racial/ethnic groups. ${ }^{3}$ Furthermore, race/ethnicity was not determined to be a significant contributor to the I-year and 10 -year survival rate of adult lung transplant recipients according to the 
2005-2009 data from the United Network for Organ Sharing (UNOS) database. ${ }^{4}$

Compared to adult lung transplant, pediatric and adolescent lung transplant have been shown to have similar survival rates. ${ }^{5}$ However, while previous studies have examined the association of race/ ethnicity on the mortality of adult lung transplant recipients, ${ }^{2-4}$ there is a lack of information on the impact of race/ethnicity on the mortality of pediatric and adolescent lung transplant recipients. We, therefore, undertook this study to understand the association between race/ethnicity and hospitalization mortality rates among pediatric and adolescent lung transplant recipients.

\section{Methods}

This was a cross-sectional study using the Nationwide Inpatient Sample (NIS) dataset from 2003-20I7. NIS is the largest all-payer inpatient care database in the United States (US), containing about 7 million (weighted, 35 million) hospitalizations each year. All pediatric and adolescent hospitalizations (i.e., in age range 0-19 years) were included in this study. NIS used the International Classification of Diseases, Ninth Revision, Clinical Modification (ICD9-CM) diagnosis and procedure codes until the third quarter of 20I5, after which it transitioned to the ICD-I0-CM format. Lung transplants were identified using ICD-9-CM diagnosis codeV42.6 and procedure codes starting with 33.5 and 33.6, and ICD-I0-CM diagnosis code Z94.2 and procedure codes starting with OBY.

An adjusted survey logistic regression model was used to assess the association between receipt of lung transplant (exposure) and inpatient mortality (outcome) by race/ethnicity. Covariates included in the study were sex, age, and primary payer. We used two-tailed hypothesis testing for all statistical analyses, and the type-I error rate was set at $5 \%$. We utilized $R$ version 3.5.I (University of Auckland,Auckland, New Zealand) and RStudio Version I I.500 I (Boston, MA) for performing all statistical analyses. The study was performed on de-identified publicly available data and was granted exempt status by the Institutional Review Board at Baylor College of Medicine, Texas, USA.

\section{Results}

During the study period (2003-2017), there were a total of 3,767 lung transplant hospitalizations among pediatric and adolescent patients, corresponding to a rate of 3.92 per 100,000 pediatric and adolescent hospitalizations in the US. Overall, the inpatient mortality rate was 3.90 per 100 lung transplant hospitalizations. Unadjusted analysis by race/ethnicity showed the highest inpatient mortality rate of $3.57 \%$ in Hispanics, followed by $3.10 \%$ in $\mathrm{NH}$-Whites and $2.19 \%$ in $\mathrm{NH}$-Blacks. Overall, when adjusted for covariates, those who received lung transplants were 8.49 times as likely to experience inpatient mortality (95\% Cl:5.66-12.73) compared to those who did not receive it. The adjusted odds of inpatient mortality were highest among Hispanics $(O R=10.11,95 \%$ $\mathrm{Cl}$ 4.93-14.95) relative to other $\mathrm{racial} / \mathrm{ethnic}$ groups; $\mathrm{NH}-$ White children and adolescents who received lung transplants were 5.45 times as likely to die in hospital (95\% Cl: 3.25-9.15) when compared with those without a transplant, whereas among $\mathrm{NH}$ Blacks, the association did not achieve statistical significance.

\section{Discussion, Conclusion, and Implications for Translation}

Overall, our study observed that among racial/ ethnic groups of pediatric and adolescent lung transplant recipients ( $\leq 19$ years of age), Hispanics had the highest inpatient mortality rate at $3.57 \%$ and significantly higher adjusted odds of inpatient mortality relative to other $\mathrm{racial} / \mathrm{ethnic}$ groups. Similar to the previous study that reported that minority adult lung transplant recipients had a higher than average index hospitalization mortality, ${ }^{2}$ we observed higher inpatient mortality in Hispanics; however, the mortality among $\mathrm{NH}$-Blacks did not achieve statistical significance ( $p$-value $>0.05)$. Sociodemographic factors may have been a significant factor in the higher inpatient mortality observed among Hispanics as a prior study determined that Hispanics had lower waitlist access to lung transplants than $\mathrm{NH}-$ Whites. ${ }^{3}$ Moreover, the observed inpatient mortality among Hispanics could also be due to possible comorbidities as diabetes was associated with early mortality among 


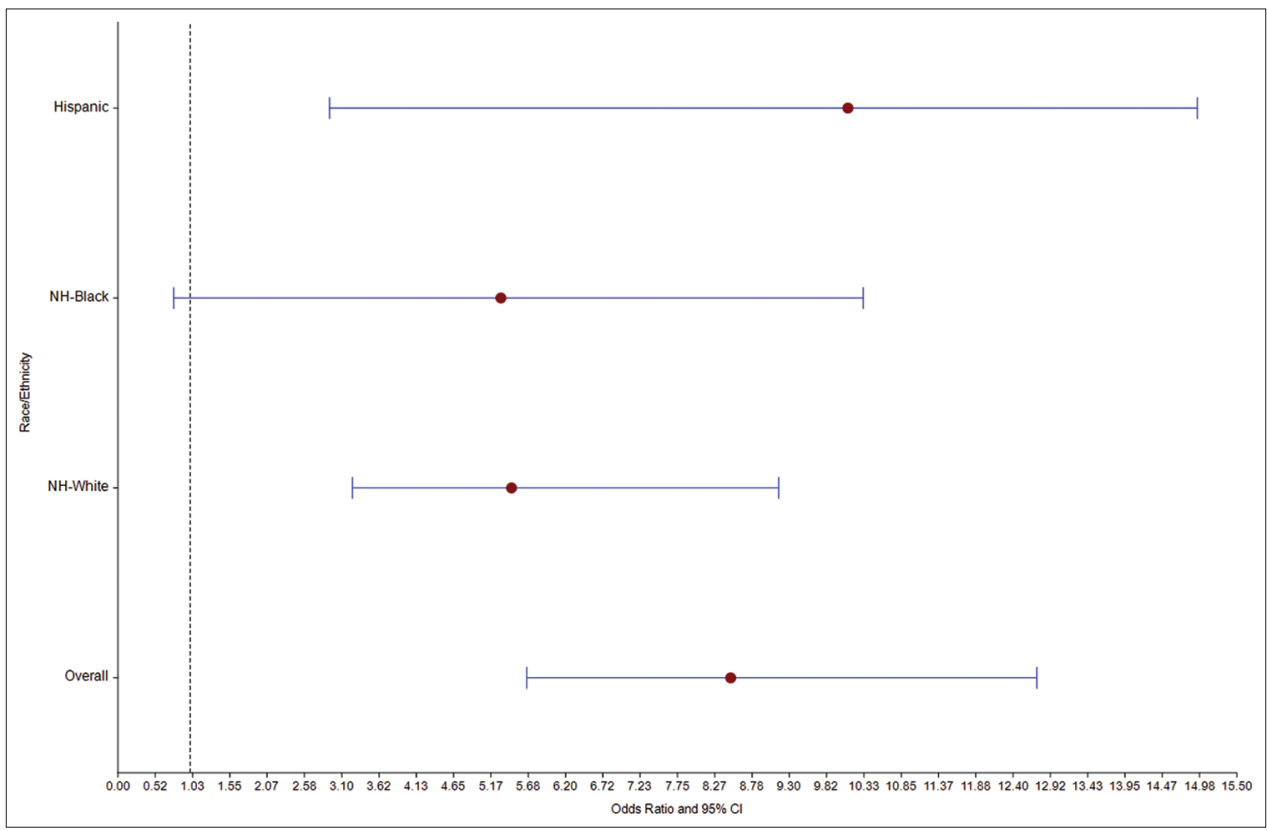

Figure I: Association Between Lung Transplant and Inpatient Mortality Among Various Racial/Ethnic Groups

lung transplant recipients in a previous study. ${ }^{4}$ Our study also found an overall increased adjusted odds of inpatient death of lung transplant recipients compared to patients who did not undergo a lung transplant. The same findings were also observed among $\mathrm{NH}$-Whites.

While our study analyzed transplant and mortality rates across racial groups, we were limited on data regarding biracial and multiracial populations because the database did not include this information. Moreover, future research into waitlist mortality and survival rates of pediatric lung transplant recipients is needed to determine if there is no difference in outcome among racial groups as was previously reported in adult lung transplant recipients. ${ }^{3,4}$ Nevertheless, from our knowledge, ours is the first and largest study in the United States that has assessed the impact of race/ethnicity on pediatric and adolescent lung transplant procedures. Therefore our study provides a foundation for future research on racial disparities in pediatric and adolescent organ transplant procedures. Our findings may guide healthcare providers when caring for pediatric and adolescent populations from different racial/ethnic backgrounds who need a lung transplant. Targeted intervention and future studies are warranted to understand the pathophysiology of increased mortality in certain races/ethnicities.

\section{Compliance With Ethical Standards}

Conflicts of Interest: None. Financial Disclosure: None. Funding/Support: None. Ethics Approval: None as the study was performed on publicly available data. Acknowledgments: None. Disclaimer: None.

\section{Key Messages}

During the study period (2003-2017), there were 3.92 lung transplants per 100,000 pediatric and adolescent hospitalizations in the US. Among those who received a lung transplant, the highest inpatient mortality rate was observed in Hispanics (3.57\%), followed by $3.10 \%$ in $\mathrm{NH}$-Whites and $2.19 \%$ in $\mathrm{NH}$-Blacks. Among Hispanics, the adjusted odds of inpatient mortality were highest (OR=10.1 I, 95\% $\mathrm{Cl}=4.93-\mathrm{I}$ 4.95) relative to other $\mathrm{racial} / \mathrm{ethnic}$ groups. 


\section{References}

I. Valapour M, Lehr CJ, Skeans MA, et al. OPTN/SRTR 2018 Annual Data Report: Lung. Am J Transplant. 2020;20 Suppl sI:427-508. doi: I0. I I I I/ajt. 15677

2. Courtwright AM, Rubin E, Robinson EM et al. In-hospital and subsequent mortality among lung transplant recipients with a prolonged initial hospitalization. Am J Transplant. 2018; I9(2):532-539. doi: I0. I I I I/ajt. I4982

3. Mooney JJ, Hedlin H, Mohabir P, Bhattacharya J, Dhillon GS. Racial and ethnic disparities in lung transplant listing and waitlist outcomes. J Heart Lung Transplant. 2018;37(3):394400. doi:10.1016/j.healun.2017.09.017

4. Jawitz OK, Raman V, Becerra D, Klapper J, Hartwig MG. Factors associated with short- versus long-term survival after lung transplant.J Thorac Cardiovasc Surg. Oct 7:S00225223(20)32699-4. doi: I0.1016/j.jtcvs.2020.09.097

5. Aurora P, Edwards LB, Christie J, et al. Registry of the International Society for Heart and Lung Transplantation:
Eleventh Official Pediatric Lung and Heart/Lung Transplantation Report-2008. J Heart Lung Transplant. 2008;27(9):978-983. doi: I0.1016/j.healun.2008.06.018

\section{PUBLISH IN THE}

International Journal of Translational Medical Reasearch and Public Health

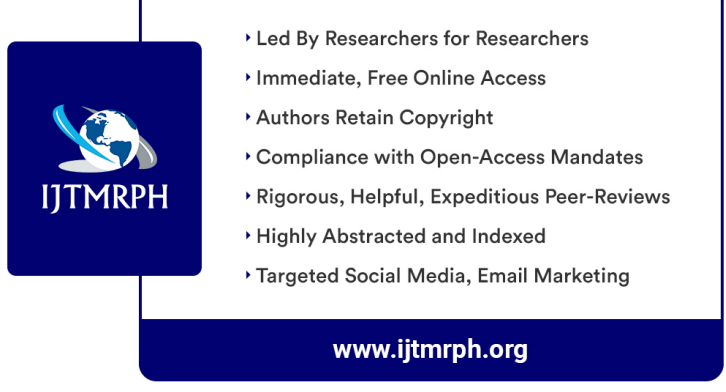

